\title{
TERTULIAS DIALÓGICAS Y ADQUISICIÓN DE COMPETENCIAS: percepción de estudiantes del grado de Educación Social
}

\section{DIALOGIC GATHERINGS AND ACQUISITION OF COMPETENCIES: the perception of Social Education students}

\author{
Cristina Laorden Gutiérrez \\ Mercedes Foncillas Beamonte
}

\begin{abstract}
Resumen
La enseñanza universitaria debe ir más allá de los conocimientos teóricos de las disciplinas. Con el objetivo de contribuir a ello proponemos las "Tertulias Pedagógicas Dialógicas" como buena práctica educativa para desarrollar competencias del grado de Educación Social. Pretendemos que el alumnado sea capaz de responder a las necesidades sociales actuales en su área de trabajo.

Tras una experiencia con 20 estudiantes de tercer curso del grado de Educación Social y la realización de un cuestionario para conocer su percepción sobre las competencias trabajadas, los resultados manifiestan una alta percepción para desarrollar mayoritariamente: el "desarrollo del compromiso ético" y la "comunicación oral", como
\end{abstract}


competencias genéricas. Y como competencias específicas destacan "identificar y emitir juicios razonados sobre posibles problemas educativos para mejorar la práctica profesional".

Otras percepciones interesantes son el aprendizaje unido a la libertad de expresión y al diálogo igualitario. Los estudiantes también destacan el nivel de aprendizaje basado en la reflexión y no en aspectos memorísticos.

\title{
Palabras Clave
}

Competencia; aprendizaje dialógico; tertulias pedagógicas dialógicas; educación social.

\begin{abstract}
University education must go beyond the theoretical knowledge of the disciplines. To contribute to this, we propose the Dialogic Pedagogical Gatherings as a good educational practice to develop competences of the Social Education degree. We want the students to be able to respond to the current social needs in their working field.

After an experience with 20 third-course students in the Social Education degree and the completion of a questionnaire to know their perception of the competences worked, the results show a high perception to develop mainly the generic competences "development of ethical commitment" and "oral communication". As specific competence, "identifying and giving informed opinions about possible educational problems to improve professional practice" is highlighted.
\end{abstract}

Other interesting perception is learning linked to freedom of expression and egalitarian dialogue. Students also emphasise the learning based on reflection and not on rote aspects.

\section{Key Words}

Competence; dialogic learning; dialogic pedagogical gatherings; social education. 


\section{Introducción}

La enseñanza universitaria debe ir más allá del conocimiento de las disciplinas. Debe profundizar sobre las formas de acercar los contenidos al mundo del alumnado, a la vida. Tenemos presente que nuestra labor dentro de la universidad es formativa lo que nos exige no sólo pensar en contenidos sino en los medios de trabajarlos dentro de las aulas (Martínez et al., 2011). La preocupación por acercar los contenidos científicos de las disciplinas a la realidad del alumnado; la preocupación por mejorar el trabajo universitario y la calidad de las intervenciones; la preocupación por contribuir a un proyecto de universidad actual, progresista, que transforme la realidad social, siempre en clave de igualdad, diálogo y justicia interesa a muchos estudiosos e investigadores (CREA, 2005; Loza, 2005; Soler y Lleras, 2003; Rekalde, Alonso Olea, Arandia , Martínez Domínguez y Zarandona, 2014).

Pero los cambios son difíciles, las estructuras universitarias siguen adaptadas a las clases magistrales donde el profesor es el protagonista del proceso de aprendizaje (Toledo, 2015) a pesar de las, cada vez más, numerosas experiencias de profesores universitarios interesados en el cambio. Ya lo señalaba Bruner, en 1997, al escribir que los avances de la educación requieren un profesorado que entienda y esté comprometido con las mejoras proyectadas. Sin este compromiso difícilmente se pueden desarrollar los planteamientos, reales y efectivos, que se señalaron no solo para la adaptación al EEES sino también a los compromisos internacionales en materia educativa y en todos los niveles. Por ello, se apela a la comunidad académica insistiendo en la necesidad de adecuar las metodologías de enseñanza y aprendizaje que favorezca este cambio (Del Pozo, 2009). Se pretende conseguir estructuras más flexibles, nuevos enfoques basados en el aprendizaje de los estudiantes y titulaciones con fuertes conexiones entre las competencias, los objetivos, los contenidos, las distintas metodologías y la evaluación que adopta el profesorado (Olmedo, 2013).

\section{Justificación}

Los cambios que se están produciendo en la universidad, describen la necesidad de proporcionar la formación necesaria para que los estudiantes respondan a las necesidades sociales y educativas del momento (Aguilera, Mendoza, Racionero y Soler, 2010). Por ello es necesario que las metodologías cambien, los contenidos se adapten a la realidad profesional y se desarrollen las competencias (Pallisera, Fullana, Planas y Del Valle, 2010). Esto es lo que Guedea (2008) denomina "profesional flexible" y supone que los estudiantes adquieran unos conocimientos técnicos y científicos, junto a una ética profesional y social, introduciendo actividades que desarrollen capacidades, habilidades o destrezas que les capaciten para razonar, tomar decisiones y comunicarse de forma eficaz (García Ruiz, 2006).

Pero debemos dar un paso más. La Unesco aprobó, en 2015, los 17 Objetivos de Desarrollo Sostenible, de cara a 2030, donde se recogen importantes metas para la educación superior. Se destaca que la educación superior es necesaria para conseguir otros objetivos no vinculados directamente con el apartado de educación pero que suponen una 
condición imprescindible como son el fin de la pobreza, la salud y bienestar, la igualdad de género, el consumo responsable, la acción por el clima o la paz, justicia e instituciones sólidas. La Unesco insiste también en el desarrollo de capacidades superiores transmisibles, cognitivas y no cognitivas, como son: resolución de problemas, pensamiento crítico, creatividad, trabajo en equipo o habilidades comunicativas que pueden aplicarse a muchos ámbitos profesionales y que también se deben tener en cuenta en la educación superior si queremos realmente contribuir a su alcance.

Trabajar estos conceptos no es posible si no conseguimos el protagonismo del estudiante. Es una necesidad no solo de aprendizaje sino también de calidad de la propia formación universitaria. Su opinión, la percepción de su propio aprendizaje y lo que le proporciona el profesorado es clave para avanzar en metodologías más alternativas y de acción. González López, Marín Díaz y Martín Fernández (2015) comprueban como, de los factores resultantes, el alumnado reconoce que ya no es solo un receptor de información y que él, con sus hábitos, afecta a la calidad de la universidad. En este sentido, la universidad ha de clarificar lo que ha de aprender el alumnado y motivar para una participación activa del mismo en su propio proceso de aprendizaje, pero también ha de secuenciar las acciones a realizar por los estudiantes y precisar el proceso evaluativo dentro de una metodología adecuada para su adquisición (Padilla y Gil, 2008; Herrero Martínez y García Viguera, 2015).

En esta línea y acercándonos a la propuesta presentada en el presente artículo, Flecha y Torrego (2012) nos recuerdan, desde la perspectiva dialógica, que el profesorado no solo transmite la cultura, sino que la vive y recrea con los demás. De esta forma se aleja de la educación bancaria tan negativa para el fomento de la reflexión, la autonomía y la investigación (Freire, 1990). Señalan que "la educación no puede cerrarse, no pueden quedarse sus decisiones en manos del grupo de "expertos" en educación: el profesorado" (Flecha y Torrego, 2012, p.22). Es preciso formar personas críticas y con planteamientos alternativos y humanizadores.

Rekalde, Alonso, Arandia, Martínez y Zarandona (2014) nos recuerdan que las funciones de los docentes se han ampliado a las de asesorar, preparar material didáctico, coordinarse con colegas, introducir nuevas tecnologías como soporte en las aulas y, sobre todo, ofrecer experiencias formativas de gran riqueza. Consideran que dentro de las aulas hay que trabajar el conocimiento y la producción y que, para dar respuesta a las nuevas tareas docentes, donde no solo se trabaje la trasmisión de conocimientos, hay que acercarse a los planteamientos comunicativos y dialógicos, lo que supone un cambio en el proceso evaluativo.

En este sentido, la universidad española se ha caracterizado por evaluar los conocimientos que el alumnado adquiría, pero en general en pocas ocasiones se evaluaba el uso que el alumnado hacía de esos conocimientos y su utilización en situaciones reales. La innovación de la evaluación es una consecuencia de la formación como desarrollo de competencias (Villardón, 2006). Así, la forma de evaluar en y por competencias implica una valoración centrada en procesos de aprendizaje (Herrero Martínez y García de Viguera 2015). En este nuevo entorno se debe terminar con el papel tradicional del profesor y el 
modelo evaluativo. En los distintos contextos se debe evaluar para obtener información e implementar acciones que conlleven una mejora del proceso educativo.

Rodríguez, Hernández y Díaz (2007) definen las competencias profesionales como la integración de conocimientos, destrezas y actitudes que permiten el desempeño profesional de calidad. Desde el punto de vista académico constituyen el resultado de un proceso de aprendizaje que deberá garantizar que los alumnos sean capaces de integrar conocimientos, habilidades, actitudes y responsabilidades que exigen los perfiles profesionales (González Maura y González Tirados, 2008). Por lo que, siguiendo a Bunk (1994), la autonomía personal y la flexibilidad en el desempeño profesional constituyen elementos esenciales. Ahora bien, conviene distinguir entre competencias genéricas o transversales, que son aquellas comunes a todos los perfiles profesionales o disciplinas, y competencias específicas, que son las que determinan un espacio profesional concreto (Pulido, 2008).

A nivel global, una competencia se alcanza al combinar una serie de atributos pertenecientes a tres categorías fundamentales: los conocimientos -componente del saber. las capacidades -componente del saber hacer-y las actitudes -componente del saber ser y estar (Leví-Orta y Ramos-Méndez, 2013). Estos autores señalan que los componentes menos favorecidos en los modelos de enseñanza- aprendizaje de los diferentes grados universitarios son los de actitudes y valores, es decir sobre los de saber ser, y que los conocimientos teóricos siguen siendo los más importantes para los profesores en algunas ramas de conocimiento.

Por todo esto, para las universidades sigue siendo un reto diseñar, por una parte, un currículo potenciador de competencias profesionales, que impliquen cambios tanto en los paradigmas de enseñanza y aprendizaje como en los roles que asumen estudiantes y profesores, y, por otra parte, concebir la formación y desarrollo de competencias genéricas y específicas en su interrelación con el proceso de formación profesional. En estas competencias genéricas se incluyen elementos de orden cognitivo y de orden motivacional, y se expresan a través de los tres tipos siguientes (González Maura y González Tirados, 2008):

- Competencias instrumentales, de orden metodológico o de procedimiento, tales como la capacidad de análisis y síntesis, de organización y planificación, y de gestión de información.

- Competencias personales, tales como la capacidad para el trabajo en equipo, la habilidad para el manejo de las relaciones interpersonales, el compromiso ético.

- Competencias sistémicas, que se manifiestan en el aprendizaje autónomo, la adaptación a nuevas situaciones, la creatividad y el liderazgo, entre otras.

En suma, las competencias serían descripciones de los aprendizajes de los estudiantes incluyendo múltiples ingredientes (recursos cognitivos, personales, sociales y de valores), implican la capacidad de movilizarlos e integrarlos, en orden a definir y responder 
adecuadamente a situaciones o problemas complejos en contextos relativamente bien delimitados, tal y como los define Escudero (2009). Supone, también, reflexionar sobre las actividades de aprendizaje y su evaluación e incorporar instrumentos y herramientas que ayuden a esta evaluación como pueden ser las exposiciones orales, debates, diarios, portfolios, dilemas éticos o proyectos de aprendizaje (Pallisera, Fullana, Planas y Del Valle, 2010).

Trabajar por competencias significa que el alumnado debe entender el aprendizaje como un circuito multidireccional donde tiene que tomar la iniciativa y estimular la capacidad crítica, ética, creativa y sensible en la gestión de su aprendizaje a todos los niveles para favorecer su formación integral. En este caso, el profesorado es un guía o un «despertador de curiosidades» que acompaña al alumnado en este trayecto (Alsina et al. 2011).

Clemente-Ricolfe y Escribá-Pérez (2012) nos presentan un interesante estudio donde se analiza la percepción de las competencias genéricas adquiridas en la universidad Politécnica de Valencia. En sus resultados se muestra que las competencias genéricas más adquiridas son la capacidad de análisis, resolución problemas y trabajo en equipo. El conjunto de las competencias genéricas se agrupó en cuatro factores: competencias metodológicas, sociales, participativas y especializadas. Las más influyentes en la percepción de los estudiantes son las metodológicas y las especializadas.

Trabajar por competencias es difícil, al igual que evaluarlas, pero no menos complejo es introducir diversas metodologías que resulten adecuadas y que faciliten su logro dentro del proceso de enseñanza-aprendizaje. Son varias las metodologías que están mostrándose adecuadas para lograr desarrollar las competencias de los universitarios. Una estrategia docente es el Aprendizaje-servicio (ApS) ya que fomenta el área social en los futuros profesionales al mismo tiempo que acerca a los estudiantes a participar en una realidad concreta como contribución a su aprendizaje y a la mejora social de su entorno real y cercano, tal y como nos propone la Estrategia Universidad 2015, que además de los aprendizajes de conocimientos científicos los estudiantes desarrollen principios éticos y valores sociales y solidarios.

Vázquez (2015) ha llevado esta metodología a su proyecto docente durante varios cursos en asignaturas como "Filosofía de la Educación" y "Pedagogía de las personas Mayores" en los grados de Pedagogía y Educación Social de la Universidad de Valencia. Ha visto como son útiles para las competencias relacionadas con el análisis crítico y la reflexión sistémica, para la toma de decisiones colaborativa y con el sentido de la responsabilidad hacia generaciones presentes y futuras concluyendo la importancia de la crítica constructiva y la reflexión en la transformación personal y social.

Otra experiencia interesante resulta un trabajo realizado sobre la competencia de comunicación oral como transversal en los estudiantes de Pedagogía y Magisterio. Fernández, Torío, Viñuela, Molina y Bermúdez (2008) lo desarrollan con debates, exposiciones, de forma interdisciplinar y trabajando en equipo para lograr que se desarrollen estas competencias dentro de las diferentes asignaturas. La utilización de foros donde los 
estudiantes generan intervenciones sobre aspectos concretos del temario, preguntas elaboradas por el profesor, situaciones sociales concretas, etc. también se consideran buenas prácticas que ayudan en el desarrollo de competencias (Correa, Pérez- Jorge y Gúzman, 2011).

Robledo, Fidalgo, Arias y Álvarez (2015) en un estudio realizado en la Universidad de León sobre la percepción de los estudiantes de educación sobre el desarrollo de competencias a través de diferentes metodologías activas, obtienen como resultado, entre otros, que uno de los métodos de aprendizaje que muestra mayor eficacia para estimular el desarrollo de competencias en la universidad es la metodología del Aprendizaje Basada en Problemas (ABP). Las razones dadas en este estudio hacen referencia a las características de esta metodología, que combina conocimientos, habilidades, actitudes y competencias dirigidas al mercado laboral. También argumentan el protagonismo del alumnado en su aprendizaje a través del trabajo autónomo y en grupos.

Unidas a las metodologías anteriores, destacamos las tertulias dialógicas como buena práctica para este desempeño y en este sentido, coincidimos con Villardón (2015), al señalarlas como propuesta metodológica para desarrollar competencia de relación humana, fundamental para todo desarrollo personal y profesional. Puesto que, como señala González Conde (2007) al hablar de competencias, la capacidad de expresar sentimientos, críticas y autocríticas va unida al trabajo en equipo o a la expresión del compromiso social o ético como competencias interpersonales importantes.

Las Tertulias Pedagógicas Dialógicas (TPD) como método de aprendizaje y buena práctica educativa, contrastada científicamente a través de proyecto INCLUD-ED (2011), nos permiten desarrollar algunas de las competencias, tanto genéricas como específicas, en los grados universitarios. En este sentido, para desarrollar las competencias necesarias de los profesionales que trabajan en equipo, el alumnado debe tener facilidad de comunicación, respeto a diversas opiniones y práctica en la escucha activa y, consideramos que, las tertulias pedagógicas dialógicas pueden contribuir a su alcance.

Un proceso metodológico dialógico lleva al alumnado a tomar conciencia real sobre su propio proceso de aprendizaje y a consolidar un pensamiento argumentativo (Arandia, Alonso-Olea y Martínez-Domínguez, 2010). De ahí, la necesidad de indagar sobre la naturaleza, los procedimientos y los efectos de este tipo de procesos. Su trabajo puede aportar algunas pistas para generar procesos educativos transformadores dentro del ámbito universitario, conectados con la sociedad (Arandia, Alonso-Olea y Martínez-Domínguez 2010). En él, la validez de las intervenciones se encuentra en relación directa con la capacidad argumentativa de las personas que interactúan, y no con las posiciones de poder que éstas puedan ocupar. El diálogo se produce entre sujetos de conocimiento que se ubican en una situación educativa entendida como acto de conocimiento, no de memorización ni de transmisión sino de problematización de la realidad que conduce a un análisis y reajuste del conocimiento en torno a la reflexión, al pensamiento crítico (Freire, 1990). Por ello, no todo diálogo tiene que ver con una relación de verdadero conocimiento como el de la relación dialógica, que implica una comunicación e intercomunicación entre personas, siempre 
abiertas a la posibilidad de conocer. El diálogo precisa, como señalábamos, de investigación crítica, y trabajar en diálogo supone contribuir a la democratización de los procesos educativos y de las organizaciones en los que éstos tienen lugar (Freire, 1990, 1997). Su estudio en las aulas universitarias nos proporciona información sobre cambios en el proceso de socialización académica, el conjunto de creencias e ideales sobre la educación y el ser educador como imaginario pedagógico, la relación profesor-alumno, la horizontalidad en el aprendizaje y la participación. Contribuye también a la reflexión sobre lo leído, a la búsqueda de significado en la realidad actual, a aprender compartiendo y a implementar un diálogo igualitario, elementos a los que el profesorado universitario está poco habituado (Foncillas y Laorden, 2014).

Las TPD aparecen cada vez más en la literatura científica como una práctica de éxito también en la educación superior. En una experiencia llevada a cabo con estudiantes de psicología en colegios de primaria, Aguilera, Prados y Gómez del Castillo (2015), nos señalan en los resultados obtenidos tras la experiencia, que los estudiantes se han sentido enriquecidos personal y profesionalmente, se han sentido con libertad, han relacionado la lectura con la vida y han aprendido de los demás al abrirse a otras realidades.

Son numerosas las experiencias en este sentido. Gabaldón, Obiol, Beltran y Benedito (2016) nos presentan una de ellas en magisterio de Infantil, Primaria y Sociología de la universidad de Valencia. Los resultados muestran la alta satisfacción de los estudiantes con esta experiencia donde se fomenta la reflexión y las vivencias con sus propias experiencias de vida. Las TPD permiten, según Fernández, Garvín y González (2012), la construcción entre todos los participantes del conocimiento, profundizando en los aspectos teóricos en relación con prácticas y vivencias de cada uno. Son estrategias de formación permanente pues contribuyen a formar una retroalimentación continua entre teoría y práctica y son un recurso didáctico en la formación de docentes. Supone una práctica que aporta sentido e ilusión y facilita la mejora, la investigación y el aprendizaje señalan estos autores.

Chocarro de Luis (2013) las presenta como modelo de saber ser y estar en el aula unido al futuro desempeño profesional. Tras una experiencia con niños de primaria en el colegio destaca que el aprendizaje de calidad emana de actividades que mueven el compromiso del alumnado, su responsabilidad y persiguen cambios en el sujeto que aprende. Este aprendizaje se puede lograr mediante experiencias reales y reflexionando sobre la práctica.

Con este artículo pretendemos mostrar, una vez más, la importancia de las TPD, en este caso, en el grado de Educación Social. Ponemos en relación la experiencia de TPD y la percepción de los estudiantes en el desarrollo de algunas de las competencias universitarias más importantes en su grado.

\section{Objetivos}


El objetivo principal es conocer la percepción de los estudiantes de $3^{\circ}$ de Educación Social en el logro de las competencias de su grado, generales y específica, a través de las TPD.

Como objetivos específicos se pretenden conocer otros elementos importantes desarrollados y vividos en las sesiones de las tertulias:

- conocer la percepción de los estudiantes tras su experiencia con TPD.

- conocer la percepción de los estudiantes con respecto al aprendizaje y su influencia en su futura vida profesional como educadores sociales tras esta práctica de éxito.

Con todo ello, esperamos poder aportar un mayor conocimiento sobre esta buena práctica como metodología dialógica, aprendizaje entre todos y la repercusión de lo aprendido en la futura vida profesional de los estudiantes.

\section{Metodología}

Esta experiencia se ha llevado a cabo en el Centro Universitario Cardenal Cisneros, adscrito a la Universidad de Alcalá, con un total de 20 estudiantes del tercer curso de grado en Educación Social. Han estado implicadas dos asignaturas del plan de estudios: Medio Escolar y Educación de Personas Adultas.

Se han utilizado dos libros para esta práctica: Carta a una maestra (1970) escrito por los Alumnos de la escuela de Barbiana y A la sombra de este árbol (1997) de Paulo Freire.

Consideramos ambos libros básicos para todo estudiante de este grado y oportunos para el tema del Aprendizaje Dialógico y las TPD como contenido curricular, ya que en ellos se recogen, teóricamente las bases de la dialogicidad.

El grupo-clase se divide en dos grupos de 10 estudiantes cada uno; agrupados en el espacio de los seminarios (pequeños grupos que se organizan en todas las asignaturas que se imparten en el centro). Las sesiones organizadas de las TPD se desarrollan cada 15 días y todas las personas integrantes de las mismas se sitúan en círculo. Previo al comienzo de las TPD, se mantuvo una reunión para explicar esta organización de los grupos, las fechas de las sesiones, decidir las normas fundamentales de respeto, puntualidad, participación y rol del moderador y, por último, seleccionar el contenido a leer de cara a la primera sesión. Se informó de la grabación de las sesiones y se firmaron las autorizaciones pertinentes.

Se llevaron a cabo 6 sesiones de lectura en cada grupo (A y B). En ambos se encontraban las dos profesoras. Ninguna de ellas actuó como moderadora y fue el alumnado quien asumió esta función, con la meta fundamental de facilitar la intervención de todo el 
alumnado. En cada sesión el grupo decidía el contenido de lectura para la siguiente existiendo un compromiso entre todos, para traer la lectura leída, subrayados los párrafos que les habían parecido más interesantes y dispuestos a participar de forma activa.

Al terminar las sesiones, los estudiantes cumplimentaron un cuestionario, elaborado por las investigadoras, en la que se han recogido distintas variables del tema a estudiar organizadas en tres bloques:

El bloque 1 se refiere a la percepción de la experiencia y contiene tres ítems: el primero referido a cómo se han sentido durante las sesiones, con 4 opciones de respuesta (muy bien, bien, regular o mal). El segundo contiene 5 afirmaciones importantes en la TPD y que los estudiantes debían valorar del 1 al 5 , siendo 1 lo menos valorado y 5 lo más valorado. Las afirmaciones son las siguientes: he compartido lectura con mis compañeros, toda opinión es válida, he podido expresarme con libertad, mi conocimiento ha aumentado con las opiniones de los demás y he estado incómodo/a. porque no me gusta hablar en público. El tercer ítem consiste en 6 elementos referidos a aspectos en la organización de las sesiones que los estudiantes valoraban del 1 al 5 , siendo también el 1 lo menos valorado y el 5 lo más valorado. Los elementos son: puntualidad, respeto mutuo, escucha, orden en las intervenciones, expresión verbal y tarea de lectura.

El bloque 2 se refiere al aprendizaje y la futura vida profesional y está formado por dos ítems con la misma escala de respuesta que la señala anteriormente del 1 al 5 . En el primero, de los ítems se señalan 6 aspectos relacionados con aprendizaje: libertad de expresión, diálogo igualitario, aprendizaje entre todos, reflexión, conocer más a los compañeros y capacidad de escucha. Todos ellos importantes en las características de las TPD. EI segundo ítem se refiere a la futura vida profesional con los siguientes elementos: aprender a escuchar, reflexionar no imponiendo tu opinión, respeto y diálogo igualitario, fomentar la transformación a través de la reflexión, participar y superar retos e interesarse por la lectura de libros clásicos de su campo profesional.

El bloque 3 se refiere a las competencias, generales y específicas. Se señalan todas las competencias de su grado universitario (anexo 1) y se debía valorar del 1 al 5 (siendo el 1 lo menos valorado y el 5 lo más valorado,) su percepción de la contribución de las TPD a su alcance.

Al tratarse de una encuesta sobre percepciones, que no ha sido diseñada para medir constructos psicológicos, no existen datos psicométricos previos sobre su fiabilidad y validez.

Para el análisis de los datos se han realizado análisis descriptivos de frecuencia y porcentajes en los diferentes elementos valorados en el instrumento descrito y utilizando el programa SPSS 23.

\section{Resultados}


A continuación, se presentan, en dos grandes categorías, los resultados más puntuados por los estudiantes, basados en los análisis descriptivos llevados a cabo, y relacionados con la adquisición de competencias del grado de Educación Social.

Categoría 1: con respecto a las competencias trabajadas.

Categoría 2: con respecto a la percepción de la experiencia y el aprendizaje y futura vida profesional.

\section{Categoría 1.- Con respecto a las competencias trabajadas.}

Se obtuvieron datos sobre la percepción del logro tanto de las competencias genéricas como de las específicas (anexo 1).

Así, el 73,7\% del alumnado del grupo, consideran que con las sesiones de TPD se desarrolla el compromiso ético en la práctica profesional, siendo ésta una de las competencias genéricas del grado de educación social objeto de estudio. En este sentido un estudiante señala, ...promueve una horizontalidad, un diálogo igualitario y respeto desde el que puedes partir para ponerte objetivos, cómo aprende la persona, acompañarla y apoyarla desde lo que ella quiere y no desde lo que tú consideras que es mejor para ella.

Otra de las competencias que perciben los estudiantes que se desarrolla con esta buena práctica es la comunicación oral $(68,5 \%)$. Perciben que les ayuda a expresarse oralmente delante de los compañeros, en cuestiones de diversa naturaleza y con contenido personal y crítico y no reproduciendo contenidos elaborados por el profesor.

Por último, el $63,2 \%$ consideran que, además, ayudan en la aplicación de los aprendizajes a la práctica y a desarrollar la capacidad creativa y emprendedora $(57,9 \%)$ (Tabla 1).

\begin{tabular}{|ll|}
\hline Competencias Generales & $\%$ \\
\hline $\begin{array}{l}\text { Desarrollar compromiso ético en } \\
\text { la práctica profesional }\end{array}$ & 73,7 \\
\hline $\begin{array}{l}\text { Desarrollar una comunicación } \\
\text { correcta oral y escrita }\end{array}$ & 68,5 \\
\hline $\begin{array}{l}\text { Aplicar los aprendizajes a la } \\
\text { práctica }\end{array}$ & 63,2 \\
\hline
\end{tabular}

Tabla 1. Percepción de las competencias trabajadas. Fuente: Elaboración propia

Con respecto a las competencias específicas (Tabla 2), resaltan, por encima de cualquier otra competencia: 
- Identificar y emitir juicios razonados sobre problemas socioeducativos para mejorar la práctica profesional $(78,9 \%)$.

-Comprender los referentes teóricos, históricos, culturales, políticos, ambientales y legales que constituyen al ser humano como protagonista de la educación $(68,4 \%)$

Estos resultados se podrían explicar debido al hecho de que durante las sesiones de tertulia se ponen en relación contenidos de diferentes asignaturas que ya han recibido y temas de carácter social, político, económico que o bien nunca han trabajado o bien, nunca han reflexionado sobre ellos.

\begin{tabular}{|c|c|}
\hline Competencias específicas & $\%$ \\
\hline $\begin{array}{l}\text { Identificar y emitir juicios razonados } \\
\text { sobre problemas }\end{array}$ & 78,9 \\
\hline $\begin{array}{l}\text { Comprender los referentes teóricos, } \\
\text { históricos, culturales, comparados, } \\
\text { políticos, ambientales y legales que } \\
\text { constituyen al ser humano como } \\
\text { protagonista de la educación }\end{array}$ & $64,8 \%$ \\
\hline
\end{tabular}

Tabla 2. Percepción de las competencias específicas. Fuente: Elaboración propia

\section{Categoría 2.- Con respecto a la percepción de la experiencia y aprendizaje y futura vida profesional.}

En base a los datos obtenidos, el $100 \%$ de los participantes señala haberse sentido bien o muy bien durante el desarrollo de las diferentes sesiones de TPD llevadas a cabo.

Según se observa en el gráfico 1 , el $52,6 \%$ considera como lo más valorado el haber compartido lectura con los compañeros/as, el $57,9 \%$ que toda opinión sea válida en estas sesiones también lo consideran muy importante y un $73,3 \%$ consideran que lo más valorado ha sido poder expresarse con libertad (ver gráfico 1). 


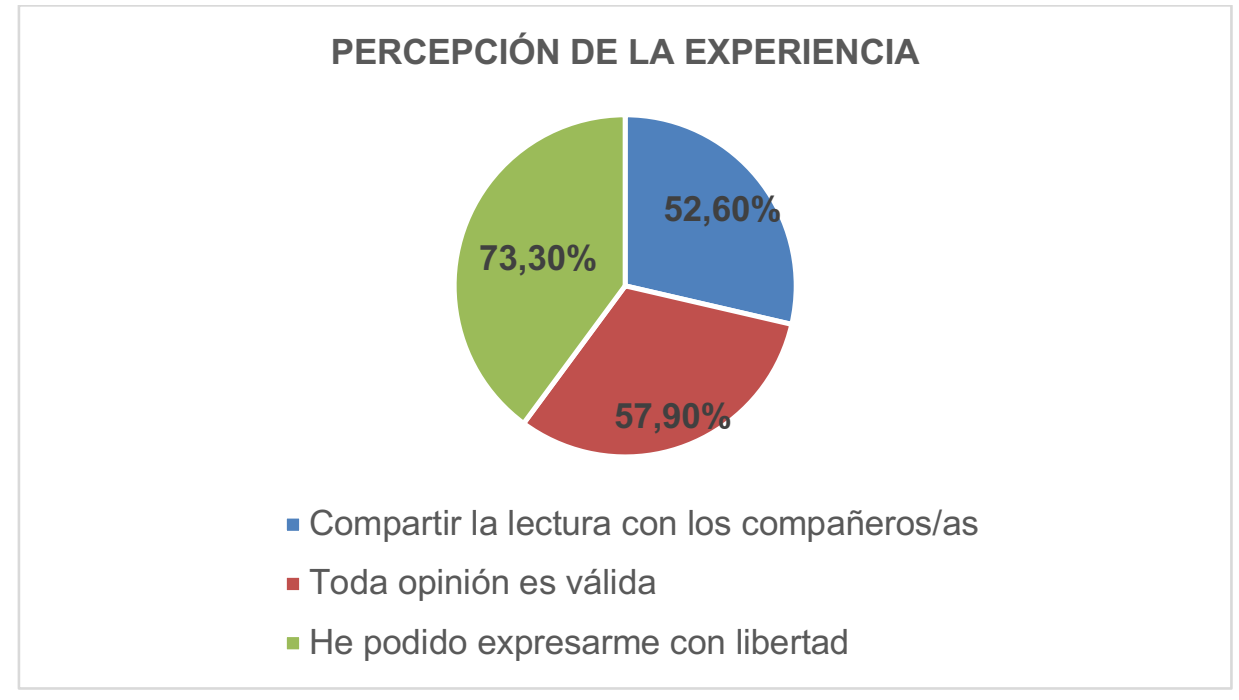

Gráfico 1

El $55 \%$ consideran como muy valorado el hecho de haber aprendido con las aportaciones de los demás.

Entre las características a valorar en el desarrollo de las sesiones se señalaron el respeto entre los miembros del grupo, la capacidad de escucha, el orden en las intervenciones, la expresión verbal y la tarea de lectura realizada. Se consideraron estas características porque suponen herramientas importantes en el desarrollo de las competencias señaladas en el grado. Los estudiantes destacan por encima de las demás las dos siguientes:

- Respeto mutuo entre los miembros del grupo $(84,2 \%)$

- Capacidad de escucha $(73,7 \%)$

Consideramos que el desarrollo de estos dos elementos supone una base importante para poder adquirir competencias generales como "trabajo en equipo" y" desarrollar una comunicación oral". En el caso de las competencias específicas ayudaría en la mediación en situaciones de riesgo y conflicto, habituales en su vida profesional.

Con respecto a su vida profesional:

La percepción de la conveniencia de conocer esta metodología con respecto a su futura vida profesional fue de un $100 \%$, argumentando esta percepción con afirmaciones como:

Sí, y además creo que es una metodología de aprendizaje que deberían conocer todos los educadores sociales, ya que se puede llevar a cabo para muchos fines, como, por ejemplo: Resolución de conflictos (...), Adquisición de habilidades sociales y aprender a respetar el turno de palabras, a escuchar a los demás (...). O ...es una propuesta interesante 
para conocer a los participantes, ya que no existen jerarquías de poder y pueden expresar sus opiniones libremente.

Podemos afirmar que facilita el logro de otra competencia específica como es "aplicar metodologías específicas a la acción educativa”. Los aspectos señalados por el alumnado fueron:

- Capacidad de escucha, lo valoraron alto y muy alto un $95 \%$.

- Respeto y Diálogo igualitario $(89,5)$

- Fomento de la transformación social y personal a través de la reflexión (52,6\%)

- Participación y superación de retos (52,6\%)

Podemos observar que la "capacidad de escucha" y el respeto" se resaltan de nuevo. Esta vez relacionada con la vida profesional por lo que suponen elementos importantes en la percepción de los propios estudiantes.

\section{Limitaciones y conclusiones}

En base a los datos presentados, podemos señalar que la metodología seguida, basada en la TPD, ha cumplido los objetivos que se habían marcado. El alumnado se ha sentido protagonista de su propio aprendizaje, ha valorado altamente haber compartido la lectura de los dos libros con el resto de sus compañeros y compañeras, así mismo ha considerado la importancia de todas y cada una de las opiniones expresadas, todas válidas y marcadas por la libertad a la hora de emitirlas. La alta percepción de la experiencia desarrollada ha contribuido a incrementar el conocimiento del alumnado. Esto se ha llevado a cabo a través de las distintas opiniones aportadas en las sesiones. Otro de los aspectos que se considera importante en el aprendizaje del alumnado es la reflexión. Se considera básica en todo proceso de aprendizaje. Tal como señala Villardón (2006) el alumnado, por medio de la reflexión, reconoce elementos que ayudan a su aprendizaje y aspectos que lo dificultan. El aprendizaje en estas circunstancias se convierte en un aprendizaje sólido, alejado del meramente memorístico. En otras experiencias anteriores, en este sentido los estudiantes han podido experimentar a través de esta buena práctica educativa que aprenden compartiendo, reflexionando a través de un diálogo igualitario alejado de las clases magistrales (Foncillas y Laorden, 2014). En definitiva, el alumnado se aleja de ser un receptor de contenidos pasivo, deja de ser vasijas, en las que se depositan conocimientos tal y como señalaba Freire (1990).

Otros datos obtenidos hacen referencia a la futura vida profesional del alumnado, siendo de destacar tanto la capacidad de escucha como el respeto, la comunicación oral y el diálogo igualitario. Aspectos estos fundamentales para profesionales de la educación social. 
Sáez Carreras (1993) sostiene que el educador social, tendrá un carácter abierto, una disposición positiva hacia la propia actividad que realiza, con capacidad para establecer lazos positivos en sus intervenciones. Por otro lado, ha de saber escuchar y respetar las ideas de los demás. Aspectos todos ellos recogidos anteriormente.

En cuanto a los resultados de las competencias que perciben que están desarrollando a través de las Tertulias Dialógicas es importante que señalen que experimentan un compromiso ético y una relación importante con la práctica. Estas ideas complementan lo señalado por autores como Arandia y Alonso (2012) cuando destacan la importancia de enseñar al alumnado calidad y profundidad en sus aprendizajes y el logro de competencias profesionales aunando teoría y práctica y, con respecto a la propia a la universidad, la importancia de permitir compromiso de responsabilidad social con los procesos de cambio y transformación educativos y buenas prácticas educativas que ayuden en la superación de la desigualdad social.

Tras presentar los resultados del estudio y considerando las aportaciones científicas trabajadas, podemos extraer una serie de conclusiones que nos ayudarán a avanzar en el trabajo y desarrollo del alcance de competencias universitarias en el grado de educación social.

La implantación de las tertulias dialógicas en el proceso de enseñanza-aprendizaje de los estudiantes del grado de educación social es muy bien percibido por los estudiantes para ayudarles a trabajar y alcanzar algunas de las competencias de su título universitario, destacando por encima de cualquier otra "desarrollar un compromiso ético" y "desarrollar una correcta comunicación oral" que a veces solo se trabaja desde las exposiciones de trabajos en clase.

Por otro lado, según los datos obtenidos, el alumnado percibe que aprende partiendo de las opiniones de sus compañeros y compañeras. No existiendo una opinión más importante que otra, todas son igual de válidas.

Los estudiantes del estudio valoran, por encima de todo en esta práctica metodológica, la importancia del respeto mutuo entre compañeros y la capacidad de escucha entre el grupo. Algo que no suele ser tan habitual en las enseñanzas universitarias tradicionales donde es el profesor el que imparte los conocimientos. Además, estos resultados ya se han encontrado en otras experiencias realizadas en cursos anteriores (Foncillas y Laorden, 2014)

Es importante señalar la utilidad que el alumnado percibe de esta práctica metodológica para implementarla en su vida profesional en un futuro próximo, acercándonos con ello al desarrollo de otros de las competencias específicas de su grado.

Este estudio presenta la limitación del número de estudiantes de la experiencia que supone una muestra pequeña, pero incluso así podemos aventurarnos a presentar esta práctica como una posible alternativa eficaz y bien percibida por los estudiantes en las innovaciones educativas en las enseñanzas universitarias. En nuestro ánimo estaría que se 
extendiera a otras asignaturas, ya que reforzaría los beneficios en el proceso de aprendizaje del alumnado y desarrollo de algunas de sus competencias.

Queda mucho trabajo por hacer, la adquisición de las competencias a lo largo de los cuatro años de estudio y la coordinación entre todas las asignaturas sería una necesidad para ir introduciendo esta buena práctica en ellas. Por otra parte, al contribuir a desarrollar competencias generales sería interesante introducir las TPD en otros grados como son Psicología, Magisterio, Trabajo Social y en general todos aquellos campos profesionales donde el trabajo en equipo, la relación con compañeros, la escucha activa y el diálogo reflexivo sea una necesidad profesional que ayude al avance y la transformación continua.

\section{Bibliografía}

Aguilera, A.; Mendoza, M.; Racionero, S. \& Soler, M. (2010). El papel de la universidad en comunidades de Aprendizaje. Revista Interuniversitaria de Formación del Profesorado, 67(24.1) 45-56.

Aguilera, A; Prados. M. \& Gómez del Castillo, $M^{a} T$ (2015). La experiencia del voluntariado universitario en las tertulias dialógicas de las comunidades de aprendizaje, REDU Revista de Docencia Universitaria, 13 (2) 249-267.

Alsina, J. (coord.). (2011). Evaluación por competencias en la universidad: las competencias transversales. Barcelona: ICE y Ediciones OCTAEDRO, S.L.

Alumnos de la escuela de Barbiana (1996). Carta a una maestra. Madrid: PPC

Arandia Loroño, M. \& Alonso-Olea, Ma . J. (2012). Universidad y comunidades de aprendizaje: aprendiendo de una relación. El caso del País Vasco. Tendencias pedagógicas, 19, 87106.

Arandia Loroño, M.; Alonso-Olea, Mª. J., \& Martínez-Domínguez, I. (2010). La metodología dialógica en las aulas universitarias. Revista de Educación, 352,309-329.

Bunk, G.P. (1994). La transmisión de competencias en la formación y perfeccionamiento profesionales de la RFA. Revista Europea de Formación Profesional. 1, 8-14.

Bruner, J. (1997). La educación puerta de la cultura. Madrid: Antonio Machado Libros.

Clemente- Ricolfe, J.S. \& Escribá-Pérez, C. (2013). Análisis de la percepción de las competencias genéricas adquiridas en la universidad. Revista de Educación, 362, 535-561.

Correa, A; Pérez-Jorge, D \& Guzmán, R (2011). El uso de herramientas de interacción dialógica asincrónica en evaluación de competencias: estudio de caso en el contexto universitario. Revista Qurriculum 24, 141-171.

Chocarro de Luis, E. (2013). Las tertulias dialógicas, un recurso didáctico en la formación de docentes. Historia y Comunicación Social, 18, 219-229.

CREA (2005). 1001 tertulias en el mundo. Barcelona: Universidad de Barcelona.

Del Pozo Andrés, M.M. (2009). El proceso de Bolonia en las aulas universitarias: una perspectiva europea. Cuestiones Pedagógicas, 19, 55-73.

Escudero Muñoz, J.M. (2009). Las competencias profesionales y la formación universitaria: posibilidades y riesgos. Revista Universitaria de Pedagogía social, 16, 65-82.

Fernández García, C Ma; Torío López, S. Ma; Viñuela Hernández, P.; Molina Martín, S \& Bermúdez Rey, T. (2008). La comunicación oral como competencia transversal de los estudiantes 
de pedagogía y magisterio: Presentación del diseño metodológico de una innovación para su trabajo en el aula. Revista de Enseñanza Universitaria, 31, 26-38.

Fernández González, S; Garvín Fernández \& González Manzanero, V (2012). Tertulias pedagógicas dialógicas: con el libro en la mano. Revista Electrónica Interuniversitaria de Formación del Profesorado, 15(4) 113-118.

Foncillas, M. \& Laorden, C. (2014). Tertulias Dialógicas en Educación Social: Transformando el Aprendizaje. International Journal of Sociology of Education, 3(3), 244-268.

Freire, P (1990), Pedagogía del oprimido. Madrid: Siglo XXI

Freire, P. (1997) A la sombra de este árbol. Barcelona: El Roure Ciencia.

Flecha, R \& Torrego, L. (2012). Aprendizaje dialógico y transformaciones sociales: más allá de los límites. Lenguaje y textos, Revista de la Sociedad española de didáctica de la Lengua y la literatura, 36, 15-24.

Gabaldón D; Obiol, S; Beltran J \& Benedito A., (2016). Tertulias dialógicas en el grado de magisterio. Congreso In-Red 2016. Congreso Nacional de Innovación Educativa y De docencia en Red. Universitat Politécnica de València.

García Ruiz, Ma R. (2006). Las competencias de los alumnos universitarios. Revista Interuniversitaria de Formación del profesorado, 20 (3) 253-26.

González Conde, J., (2007). El concepto de competencia y su aplicación a la formación de profesores. En R Ma Ávila Ruiz. R. López Atxurra, R. \& E. Fernández de Larrea (ed.). Las competencias profesionales para la enseñanza-aprendizaje de las Ciencias Sociales ante el reto europeo y la globalización. Bilbao: Ed. Asociación Universitaria de profesores de Didáctica de las Ciencias Sociales.

González López, I., Marín Díaz, V. \& Martín Fernández, MªA. (2015). El significado de la Universidad desde la mirada del alumnado. Indicadores para su evaluación. Aula de Encuentro, 17, (2) 33-58.

González Maura, V. \& González Tirados, RMa . (2008). Competencias Genéricas y Formación Profesional; Un análisis desde la docencia universitaria. Revista Iberoamericana de Educación, 47, 185-209.

Guedea Medrano, I. (2008). Las demandas de las empresas en competencias genéricas y transversales de los titulados universitarios. ICE Universidad de Zaragoza.

Herrero Martínez, R M ${ }^{a}$ \& García de Viguera, $M^{\mathrm{a} C}$ C. (2015). Planificación de la evaluación por competencias con el alumnado de educación superior. Aula de Encuentro, 17, (2) 127154.

INCLUD-ED Consortium (2011). Actuaciones de éxito en las escuelas europeas. Madrid: MEC. Recuperado de http://www.nesetweb.eu/sites/default/files/actuaciones-de-exito-en-lasescuelas-europeas.pdf

Leví-Orta, G.C. \& Ramos-Méndez, E. (2013). Componentes de las competencias en los nuevos grados de algunas universidades españolas Revista de Educación, 362, 623-658.

Loza Aguirre, M. (2005). Tertulias literarias. Cuadernos de pedagogía, 341, 66-69

Martínez, I; Arandia, M.T.; Alonso, M.J.; Del Castillo, L.; Zarandona; E. \& Rekalde, I. (2011). Trabajar con Metodologías participativas en la formación universitaria, todo un desafío. Investigación en la Escuela, 75, 101-113.

Olmedo Moreno, E. Ma (2013). Enfoques de aprendizaje de los estudiantes y metodología docente. Evolución hacia el nuevo sistema de formación e interacción propuesta en el EEES. Revista de Investigación Educativa, 31 (2) 411-429. 
Padilla, M.T. \& Gil, J. (2008). La evaluación al aprendizaje en la educación superior: condiciones y estrategias para su aplicación en la docencia universitaria. Revista Española de Pedagogía. 241, 267-485.

Pallisera Díaz, M.; Fullana Noell, J.; Planas Lladó, A. \& Del Valle Gómez, A. (2010) La adaptación al espacio europeo de educación superior en España. Revista Iberoamericana de Educación 52(4) ,1-13.

Pulido Trullén, J.I. (2008). Competencias Genérica ¿qué son? En Competencias genéricas y transversales de los titulados universitarios. ICE Universidad de Zaragoza.

Rekalde Rodríguez, I; Alonso Olea, J.; Arandia Loroño, M.; Martínez Domínguez, I. \& Zarandona De Juanes, E. (2014). Las tertulias literarias dialógicas en los procesos de enseñanza universitarios: Reflexiones desde la práctica docente. Revista de Formación e Innovación Educativa Universitaria. 7 (3) 155-172.

Robledo, P.; Fidalgo, R.; Arias, O. \& Álvarez, Mª L. (2015). Percepción de los estudiantes sobre el desarrollo de competencias a través de diferentes metodologías activas. Revista de Investigación Educativa, 33(2), 369-383.

Rodríguez González, R.; Hernández Nanclares, N \& Díaz Fondón, $M^{a} A$ A. (2007). Cómo planificar asignaturas para el aprendizaje de competencias. Documentos ICE. Universidad de Oviedo.

Saez Carreras, J. (1993). El educador social. Murcia: Universidad de Murcia.

Soler, M. \& Lleras, J. (2003). Las tertulias Literarias Dialógicas; Compartiendo lectura y cultura. CREFAL Revista Decisió, 6, 24-28.

Toledo Lara, G. (2015). La universidad española y el proceso de Bolonia: consideraciones para su análisis. Revista Electrónica Actualidades Investigativas en Educación ,15(2), 1-24.

UNESCO (2017). La Educación para el desarrollo sostenible (recuperado de www.unesco.org/gemreport

Vázquez Verdera, V. (2015). El aprendizaje-servicio: una estrategia para la formación de competencias en sostenibilidad. Foro de Educación, 13(19), 193-212.

Villardón Gallego, L. (2006). Evaluación del aprendizaje para promover del desarrollo de competencias. Educación siglo XXI, 24, 57-76.

Villardón Gallego, L. (coord.) (2015). Competencias genéricas en educación superior: Metodologías específicas para su desarrollo. Madrid: Narcea

\section{ANEXO 1}

Competencias generales y específicas del Grado de Educación social de la Universidad de Alcalá

\section{Competencias Generales}

Desarrollar compromiso ético en la práctica profesional 


\begin{tabular}{|l|}
\hline Trabajar en Equipo \\
\hline Aplicar los aprendizajes a la práctica \\
\hline Desarrollar la capacidad creativa y emprendedora \\
\hline Utilizar y evaluar las TIC con fines formativos \\
\hline Desarrollar una comunicación correcta oral y escrita \\
\hline
\end{tabular}

\section{Competencias Específicas}

Comprender los referentes teóricos, históricos, culturales, comparados, políticos, ambientales y legales que constituyen al ser humano como protagonista de la educación

Diagnosticar situaciones complejas que fundamenten el desarrollo de las acciones socioeducativas.

Diseñar planes, programas, proyectos y actividades de intervención socioeducativa en diversos contextos

Aplicar metodologías específicas a la acción educativa

Formar agentes de intervención socioeducativa y comunitaria

Elaborar e interpretar informes técnicos, de investigación y evaluación sobre acciones, procesos y resultados socioeducativos

Identificar y emitir juicios razonados sobre problemas

Elaborar y gestionar medios y recursos...

Comprender la trayectoria de la Educación Social

Diseñar y desarrollar procesos de participación...

Elaborar y gestionar medios y recursos para la acción socioeducativa

Intervenir en proyectos y servicios socioeducativos...

Promover procesos de dinamización cultural y social

Mediar en situaciones de riesgo y conflicto

Gestionar y coordinar entidades, equipamientos y grupos...

Dirigir, coordinar y supervisar planes, programas y proyectos socioeducativos 
Asesorar y acompañar a individuos y grupos en procesos de desarrollo socioeducativo

Realizar estudios prospectivos y evaluativos sobre características, necesidades y demandas socioeducativas.

Referencia bibliográfica:

Laorden, C. y Foncillas, M.: (2019) Tertulias dialógicas y adquisición de competencias: percepción de estudiantes del grado de educación social. Aula de Encuentro, $n^{\circ} 21$, volumen 1, pp. 40-59

Cristina Laorden Gutiérrez Profesora Titular del Departamento de Educación y Psicología del CU Cardenal Cisneros, adscrito a la Universidad de Alcalá Correo-e: cristina.laorden@cardenalcisneros.es

Mercedes Foncillas Beamonte Profesora Titular del Departamento de Educación y Psicología del CU Cardenal Cisneros, adscrito a la Universidad de Alcalá Correo-e: mercedes.foncillas@cardenalcisneros.es 